\title{
Publisher Correction: Aspartate is a limiting metabolite for cancer cell proliferation under hypoxia and in tumours
}

Javier Garcia-Bermudez, Lou Baudrier, Konnor La, Xiphias Ge Zhu, Justine Fidelin, Vladislav O. Sviderskiy, Thales Papagiannakopoulos, Henrik Molina, Matija Snuderl, Caroline A. Lewis (D), Richard L. Possemato (D) and Kıvanç Birsoy (D)

Correction to: Nature Cell Biology https://doi.org/10.1038/s41556-018-0118-z, published online 25 June 2018.

In the version of this Letter originally published, the competing interests statement was missing. The authors declare no competing interests; this statement has now been added in all online versions of the Letter.

Published online: 8 August 2018

https://doi.org/10.1038/s41556-018-0184-2

\section{Publisher Correction: AMP kinase promotes glioblastoma bioenergetics and tumour growth}

Rishi Raj Chhipa, Qiang Fan, Jane Anderson, Ranjithmenon Muraleedharan, Yan Huang, Georgianne Ciraolo, Xiaoting Chen, Ronald Waclaw, Lionel M. Chow (D), Zaza Khuchua, Matthew Kofron, Matthew T. Weirauch (D), Ady Kendler, Christopher McPherson, Nancy Ratner, Ichiro Nakano, Nupur Dasgupta, Kakajan Komurov and Biplab Dasgupta iD

Correction to: Nature Cell Biology https://doi.org/10.1038/s41556-018-0126-Z, published online 18 June 2018.

In the version of this Article originally published, the competing interests statement was missing. The authors declare no competing interests; this statement has now been added in all online versions of the Article.

\section{Publisher Correction: IRE1 $\alpha$ governs cytoskeleton remodelling and cell migration through a direct interaction with filamin $A$}

Hery Urra, Daniel R. Henriquez, José Cánovas, David Villarroel-Campos, Amado Carreras-Sureda, Eduardo Pulgar, Emiliano Molina, Younis M. Hazari, Celia M. Limia, Sebastián Alvarez-Rojas, Ricardo Figueroa, Rene L. Vidal, Diego A. Rodriguez, Claudia A. Rivera, Felipe A. Court, Andrés Couve, Ling Qi, Eric Chevet, Ryoko Akai, Takao Iwawaki, Miguel L. Concha, Álvaro Glavic, Christian Gonzalez-Billault and Claudio Hetz (D)

Correction to: Nature Cell Biology https://doi.org/10.1038/s41556-018-0141-0, published online 16 July 2018.

In the version of this Article originally published, the competing interests statement was missing. The authors declare no competing interests; this statement has now been added in all online versions of the Article. 